\title{
A PRAXIS DA FISIOTERAPIA NO PROGRAMA HIPERDIA: CONCEITOS E DIRETRIZES
}

\section{THE PHYSIOTERAPY PRAXIS AT PROGRAMA HIPERDIA: CONCEPTS AND GUIDELINES}

\author{
Bruna Evlyn Hack ${ }^{1}$, Bruna Rodrigues Nakajima ${ }^{1}$, Marcelo Taglietti \\ ${ }^{1}$ Graduada em Fisioterapia, Centro Universitário Assis Gurgacz (FAG), ${ }^{2}$ Fisioterapeuta Doutor, \\ Docente do curso de fisioterapia do Centro Universitário Assis Gurgacz (FAG). \\ *Autor correspondente: mtaglietti@fag.edu.br, https://orcid.org/0000-0003-3650-3905
}

DOI: $10.35984 / f j h . v 2 i 1.185$

\section{RESUMO}

Introdução: Atualmente, a hipertensão arterial e a diabetes mellitus constituem a principal causa de morbimortalidade na população brasileira. Dessa forma, é necessária a elaboração de ações direcionadas a contornar essa grave situação de saúde pública, criando estratégias para promoção e prevenção. O programa Hiperdia é destinado ao cadastramento e acompanhamento dos portadores de doenças crônicas não transmissíveis, promovendo orientações e promoção de saúde. Objetivo: identificar as possíveis atuações do fisioterapeuta dentro do programa de saúde pública Hiperdia, criar propostas mais específicas, com diretrizes de atuação profissional. Método: O estudo trata- se de uma revisão interativa que aconteceu a partir de uma busca detalhada, realizada nas bases de dados: Scientific Electronic Library Online (Scielo), Medical Literature Analysis and Retrieval System Online (MedLine /PubMed) e sites do Ministério da Saúde. Resultados: Inegável é a necessidade de se investir na prevenção destas doenças crônicas e a participação do fisioterapeuta pode ser benéfica neste contexto. Conclusão: Portanto, em virtude do tamanho modesto de materiais encontrados abordando a atuação do fisioterapeuta no programa Hiperdia, é importante enfatizar a necessidade de mais estudos abordando o tema, visando esclarecer o papel do profissional em cada nível de atenção à saúde.

Palavras-chave: Fisioterapia, Hipertensão arterial e Diabetes.

\begin{abstract}
Introduction: Currently, hypertension and diabetes mellitus are the main cause of morbidity and mortality in the Brazilian population. Thus, it is necessary to develop actions aimed at circumventing this serious public health situation, creating strategies for promotion and prevention. The Hiperdia program is designed to register and monitor patients with chronic non-communicable diseases, promoting guidance and health promotion. Objective: to identify the possible actions of the physiotherapist within the Hiperdia public health program, to create more specific proposals, with guidelines for professional practice. Method: The study is an interactive review that happened from a detailed search, carried out in the databases: Scientific Electronic Library Online (Scielo), Medical Literature Analysis and Retrieval System Online (MedLine / PubMed) and Ministry websites Health. Results: Undeniable is the need to invest in the prevention of these chronic diseases and the participation of the physiotherapist can be beneficial in this context. Conclusion: Therefore, due to the modest size of materials found addressing the performance of the physiotherapist in the Hiperdia program, it is important to emphasize the need for further studies addressing the topic, aiming to clarify the role of the professional at each level of health care.
\end{abstract}

Keywords: Physiotherapy, Hypertension and Diabetes. 


\section{INTRODUÇÃO}

Atualmente, a hipertensão arterial e a diabetes mellitus constituem a principal causa de morbimortalidade na população brasileira. Segundo a pesquisa do IBGE, realizada em 2003, elas estão entre as doenças crônicas que mais acometem a população, em torno de $29,9 \%$ dos brasileiros. Dessa forma, é necessária a elaboração de ações direcionadas a contornar essa grave situação de saúde pública, buscando a reorganização da atenção básica, criando estratégias para promoção e prevenção das complicações, favorecendo uma melhor qualidade de vida (MINISTÉRIO DA SAÚDE, 2001; IBGE, 2013).

O programa hiperdia é destinado ao cadastramento e acompanhamento dos portadores de doenças crônicas não transmissíveis, promovendo orientações e atuando por meio de estratégias para prevenção e promoção da saúde. O programa é realizado nas unidades básicas de saúde e conta com uma equipe multidisciplinar composta por, um médico, um enfermeiro, um a dois auxiliares de enfermagem e quatro a seis agentes de saúde. Contudo o profissional fisioterapeuta vem lutando por mais espaço dentro do programa, mas sua atuação ainda é pouco abordada na literatura (PORTAL DA SAÚDE, 2019).

Junto dessa realidade de altos índices de portadores da DM e HAS na população brasileira e as inúmeras incapacidades que provocam, o hiperdia pretende uma reabilitação psicológica, física e social dos portadores. Surge então a necessidade de entender melhor o papel do fisioterapeuta dentro do programa e suas possíveis atribuições (MINISTÉRIO DA SAÚDE, 2001). O objetivo do presente estudo é identificar as possíveis atuações do fisioterapeuta dentro do programa de saúde pública Hiperdia.

\section{METODOLOGIA}

Trata- se de uma revisão integrativa de literatura que aborda a temática de atuação do fisioterapeuta no programa hiperdia. A busca dos artigos envolvendo a participação do fisioterapeuta no programa de saúde público hiperdia, aconteceu a partir de uma busca detalhada, realizada nas bases de dados: Scientific Electronic Library Online (Scielo), Medical Literature Analysis and Retrieval System Online (MedLine /PubMed) e sites do Ministério da Saúde. Os artigos foram obtidos por meio dos descritores: Fisioterapia, Hipertensão arterial e Diabetes, as mesmas foram verificadas na plataforma Decs. A pesquisa de referências se restringiu a artigos publicados entre 2001 a 2019. Escritos apenas na língua portuguesa. Sendo que a última busca feita aconteceu em maio de 2019. Os artigos inseridos no estudo foram relacionados com base no programa de saúde pública hiperdia e a atuação do profissional fisioterapeuta nos diferentes níveis de atenção prestados.

Foram incluídas publicações referentes ao assunto baseado no conceito principal a atuação do fisioterapeuta como possível integrante do programa hiperdia. Como critérios de exclusão, foram descartados os artigos que não trabalhassem o tema sugerido, não redigido na língua portuguesa e os que não foram publicados dentro dos anos estipulados. Após o levantamento das publicações, foi então realizada a leitura e analise dos títulos e resumos segundo os critérios de inclusão e exclusão. A avaliação da qualidade dos artigos utilizados foi verificada na plataforma QUALIS. Os demais itens analisados foram conteúdos de confiabilidade, visto que, integram a base de dados do Ministério da Saúde e Cadernos de Atenção Básica. 


\section{RESULTADOS}

O programa hiperdia é destinado ao cadastramento e acompanhamento de portadores de duas doenças crônicas, a hipertensão arterial e/ou a diabetes mellitus. Este acompanhamento acontece através da rede ambulatorial do Sistema Único de Saúde (SUS), permitindo ao usuário assistência integral. Dessa forma, permite ao sistema, conhecer o perfil epidemiológico desta população, realizar o cadastramento e a partir deste, acompanha a situação dos portadores, orientar e desenvolver estratégias para prevenção e intervenção (PORTAL DA SAÚDE, 2011).

Inegável é a necessidade de se investir na promoção de saúde e prevenção destas doenças crônicas, que atualmente constituem a principal causa de morbimortalidade no Brasil. Além de influenciar negativamente a qualidade de vida, com consequências físicas, psicológicas e sociais, geram inúmeros problemas pela grande incidência de complicações, responsáveis por altos custos ao sistema de saúde pública (AMORIM SERPA; DANTAS DE LIMA; DORNELAS DA SILVA, 2018).

Baseado neste quadro, o Ministério da Saúde, em conjunto as sociedades científicas (Cardiologia, Diabetes, Hipertensão e Nefrologia), as federações nacionais dos portadores, as secretarias estaduais, através do CONASS, e as secretarias municipais de saúde, através do CONASEMS, apresenta o Plano de Reorganização da Atenção a Hipertensão Arterial e Diabetes mellitus, o Hiperdia (MINISTÉRIO DA SAÚDE, 2001).

Com o intuito de alcançar ações mais efetivas e voltadas a grandes grupos populacionais, é indispensável à integração entre os diversos níveis de atenção no sistema de saúde. Visando ações na prevenção, proteção, promoção e vigilância, além de, oferecer assistência voltada aos grupos e às necessidades individuais (MINISTÉRIO DA SAÚDE, 2008).

\section{Fluxograma do programa:}

Depois de realizado o cadastramento no Hiperdia, o acompanhamento dos usuários é a participação no grupo operativo. O mesmo deve ser realizado pelos profissionais integrantes do programa na atenção básica, através de encontros semanais, quinzenais ou mensais, para que os participantes das atividades recebam as medicações prescritas, em algumas unidades, os coordenadores utilizam rodas de conversa e atividades dinâmicas para tornar os encontros mais envolventes e estimular a participação dos usuários. (NOGUEIRA, 2013)

A fim de garantir uma atenção integral aos portadores de diabetes mellitus e/ou hipertensão arterial, é necessário o acompanhamento na unidade básica de saúde e se necessário, em unidades de referência secundária e terciária. Os pacientes estáveis e com andamento satisfatório no programa, podem ser avaliados em intervalos de três a quatro meses, pela equipe multidisciplinar. A recomendação é que essas reavaliações ocorram a cada três meses (MINISTERIO DA SAUDE 2001).

\section{Diretrizes do programa:}

O programa visa o fortalecimento dos Sistemas de Vigilância em Saúde para o Cuidado Integral de doenças crônicas não transmissíveis. Com isso, permitir a criação de um perfil dessa população, incluindo os danos e os problemas de saúde, seja estes de competência do setor saúde ou de outros setores. E através desta análise, tornase possível a criação de estratégias e ações, para uma atuação mais efetiva, com foco nas reais necessidades dos usuários (DUARTE, 2003). 
A coleta de dados se torna essencial no processo, sendo base de para a compreensão dos problemas de saúde, possibilitando a tomada de decisões quanto às aplicações e investimentos em qualificação da equipe, tecnologia, instrumentação, modernização e informatização. Os dados são direcionados aos serviços de saúde tais como serviços ambulatoriais e hospitalares, quantificando dados de morbidade e mortalidade e fatores de risco (VIACAVA, 2002). É importante evidenciar que a produção dessas estatísticas é baseada em dados epidemiológicos e sociodemográficos. De forma geral, o Sistema de Acompanhamento de Hipertensos e Diabéticos, possibilita o monitoramento de determinadas populações de risco, 0 planejamento de ações e o cálculo do consumo de medicamentos (MALTA ET al., 2006).

\section{Atribuições governamentais:}

O Sistema Único de Saúde (SUS) fornece um acesso integral, gratuito a toda a população e atua nos três níveis de atenção. É um direito de todos, visando à prevenção, promoção e reabilitação. As ações e serviços de saúde tem sua gestão de forma hierarquizada, que acontece entre três categorias da Federação: a União, os Estados e os municípios. A hierarquização deve atuar dentro dos níveis de atenção, garantindo formas de acesso a serviços que façam parte da complexidade requerida pelo caso, recursos disponíveis numa determinada região.

O município assume responsabilidades e recebe recursos destinados a atenção básica de saúde, estando apto a encaminhar de acordo com o nível de complexidade exigida. O estado possui quatro atribuições básicas, sendo elas, a gestão do SUS no âmbito estadual, o incentivo a atenção integral pelos municípios, atuação de caráter transitório em populações ainda não assumidas pelos seus próprios municípios e a promove a harmonização, integração e modernização dos sistemas municipais. Quanto à atuação federal, são destacadas quatro funções, sendo elas, a gestão do SUS no âmbito nacional, o incentivo da gestão municipal e estadual, a harmonização, integração e modernização dos sistemas estaduais, além da normalização e coordenação à gestão nacional do SUS.

$O$ atendimento no SUS acontece em três níveis de atenção, na atenção primária, o enfoque está na educação e prevenção, sendo ela o primeiro contato entre o usuário e o sistema, neste nível estão as Unidades Básicas de Saúde, onde são realizados procedimentos de baixa complexidade. $\mathrm{Na}$ atenção secundária os procedimentos alcançam média complexidade, sendo que as intervenções acontecem em clínicas, Unidades de Pronto Atendimento e hospitais. No terceiro nível de atenção, está direcionada aos casos de alta complexidade, que implicam manobras mais invasivas e que oferecem risco a vida, estes, ocorrem nos hospitais de grande porte.

\section{Objetivos do programa:}

O programa determina estimular os profissionais envolvidos na atenção básica, para que promovam medidas coletivas na prevenção primária, na identificação da necessidade de encaminhamento, a uma atenção secundária e/ou terciária e suas complicações. Dessa forma, detectar, estabelecer diagnóstico, identificar lesões, complicações crônicas e efetuar tratamento adequado para a HAS e o DM, caracterizam-se como um verdadeiro desafio para o Sistema Único de Saúde. Pois são situações que necessitam de uma intervenção imediata, não somente pela alta prevalência na população brasileira, como também pelo alto grau de incapacidade que 
provocam. O hiperdia busca a reabilitação psicológica, física e social dos portadores dessas patologias. (MINISTERIO DA SAÚDE, 2001; AMORIM SERPA; DANTAS DE LIMA; DORNELAS DA SILVA, 2018).

\section{Equipe de trabalho:}

A equipe é composta por um médico, um enfermeiro, um a dois auxiliares de enfermagem e 4 a 6 agentes de saúde, os quais devem atuar de forma integrada e com níveis de competência bem estabelecidos na abordagem da hipertensão arterial e do diabetes mellitus (MINISTÉRIO DA SAÚDE, 2001).

O médico é responsável por identificar fatores de risco, realizar consultas, confirmar o diagnóstico, solicitar exames, prescrever a medicação, quando necessário, encaminhar para unidades de referência secundária e terciária e juntamente da equipe, programar estratégias para educação do paciente. 0 enfermeiro tem a função de capacitar os auxiliares de enfermagem e os agentes comunitários e supervisionar suas atividades, realizar consultas, encaminhar ao tratamento médico quando necessário, desenvolver atividades educativas de promoção de saúde e estabelecer junto com a equipe estratégias que possam favorecer a adesão dos grupos de risco (MINISTÉRIO DA SAÚDE, 2001).

$\mathrm{O}$ auxiliar de enfermagem verifica sinais vitais, peso, altura e circunferência abdominal, orienta sobre a mudança de hábitos, realiza o agendamento de consultas e reconsultas, faz o controle dos equipamentos e fornece medicamentos, orienta sobre a automonitorização (glicosúria e glicemia capilar) e técnica de aplicação de insulina. Os agentes comunitários de saúde esclarecem a comunidade sobre medidas de prevenção, realizam a identificação na população em geral, portadores dos fatores de risco e encaminham os mesmos, à unidade de saúde, onde é realizadas orientações sobre dieta, atividades físicas, controle de peso, mudança nos hábitos de vida diária (MINISTÉRIO DA SAÚDE, 2001).

Atualmente a inserção de outros profissionais em especial o fisioterapeuta, nutricionista, assistente social e educador físico, vem sendo amplamente discutida, considerando que a inserção destes profissionais pode ser benéfica no sentido de atender de forma mais completa as necessidades apresentadas, visando saúde e qualidade de vida dos usuários do programa hiperdia (MINISTÉRIO DA SAÚDE, 2001).

\section{Atribuições do fisioterapeuta:}

O fisioterapeuta nos grupos de hiperdia pode contribuir com um olhar diferenciado, integral, uma vez que compreende o indivíduo como um ser funcional. A atuação determina orientação e educação da população, através de propostas de ações coletivas e individuais, a elaboração de projetos, que propicie assistência integral. Visando a prevenção de distúrbios cinético funcionais em todas as etapas da vida, realiza o tratamento e a reabilitação além de atendimentos domiciliares quando necessário (BATISTA SILVANO, 2018).

As ações englobam o levantamento epidemiológico, o fornecimento de orientações, a prescrição e conduta de tratamentos, o planejamento e a execução de ações terapêuticas, o treinamento de outros profissionais quanto a abordagens, atua na prevenção da complexidade dos quadros crônicos, reduzindo os gastos públicos, restringindo os danos e sequelas já instaladas, através de ações terapêuticas e preventivas (BATISTA SILVANO, 2018). 


\section{Fisioterapeuta nos níveis primário, secundário e terciário:}

A fisioterapia possuía apenas caráter reabilitativo, com enfoque na doença e não na prevenção. Hoje, ressalta-se a importância da inclusão do fisioterapeuta, não restrito apenas ao caráter reabilitador, mas também como promotor de saúde e atuante na prevenção dentro dos programas de saúde pública (BATISTA SILVANO, 2018).

No nível de atenção primária as ações são voltadas á educação, através de orientações, realização de eventos e palestras com a comunidade, destinados a promoção de saúde, prevenindo doenças e danos permanentes ou temporários (BATISTA SILVANO, 2018). Neste âmbito, são ofertadas dinâmicas em grupo, com atividades socioeducativas e criando através destas, construções mutuas. Os profissionais da equipe, através do cotidiano, compreendem as necessidades não só do usuário, mas também do cuidador (SALES, 2016).

$\mathrm{Na}$ atenção secundária, cabe ao fisioterapeuta à prestação de serviços dentro do âmbito de consultórios, centros reabilitativos e em hospitais, sendo que a sua atuação entra na prescrição de diagnósticos, solicitação de exames de imagem complementares ao diagnóstico, planejamento e prescrição de projetos, além do estabelecimento de uma rotina de assistência fisioterapêutica dentro do programa hiperdia (BATISTA SILVANO, 2018).

A ação a nível terciário tem caráter reabilitativo, onde concentra sua atuação no controle e manutenção das doenças, reabilitando as sequelas já instaladas, com o objetivo de atingir uma recuperação completa e reintegração do individuo na sociedade (JÚNIOR, 2007).

Existe uma relação de interdependência entre os fisioterapeutas e os outros profissionais da saúde dentro do programa, visando elevar a qualidade de vida do indivíduo. A inserção do fisioterapeuta nos diversos níveis de atenção fornece uma nova redefinição da práxis profissional, proporcionando maior possibilidade de atuação em cada etapa de intervenção (JÚNIOR, 2007).

\section{Patologias:}

A diabete mellitus e a hipertensão arterial são doenças crônicas, prevalentes, com altos custos sociais, que se mostram em números crescentes, representando um importante problema social hoje (AMORIM SERPA; DANTAS DE LIMA; DORNELAS DA SILVA, 2018).

A Diabete mellitus é uma síndrome metabólica, resultante da redução nos níveis de insulina e/ou da incapacidade da mesma de exercer adequadamente suas funções. É caracterizada por hiperglicemia crônica, que no decorrer do tempo pode acarretar na disfunção e falência dos órgãos envolvidos. A classificação é baseada na dependência de insulina ou na independência dela. A diabetes mellitus tipo 1, é desencadeada primariamente da destruição de células beta pancreáticas com tendência cetoacidose. Já a diabetes tipo 2, acontece por meio da resistência e deficiência relativa na secreção de insulina (MINISTÉRIO DA SAÚDE, 2001).

A hipertensão arterial se refere a uma pressão arterial sistólica maior ou igual a 140 mmhg e uma pressão arterial diastólica também maior ou igual a 90 mmhg, sem o uso de medicação. É uma condição clínica, multifatorial, que mantem o portador em altos níveis de pressão arterial. É comumente associada a alterações na estrutura e função dos órgãos envolvidos, com foco especial no coração, encéfalo, rins e vasos 
sanguíneos, ocorrem alterações no metabolismo, elevando os riscos de possíveis acidentes cardiovasculares e até mesmo fatais (MINISTÉRIO DA SAÚDE, 2001).

As duas doenças são abordadas de forma conjunta no programa pela apresentação dos fatores comuns entre ambas, tais como a etiopatogenia, os fatores de risco, a cronicidade, além da necessidade de controle permanente (MINISTÉRIO DA SAÚDE, 2001).

\section{Ações de promoção à saúde:}

A promoção à saúde relacionada às doenças crônicas é realizada por meio de uma equipe multidisciplinar, onde o fisioterapeuta, como parte do programa hiperdia, pode atua através de ações em palestras, dinâmicas e eventos com a comunidade, com atividades práticas em grupo ou individuais, como também, orienta e disponibiliza materiais informativos no sentido a educar positivamente quanto à adoção de hábitos saudáveis (BATISTA SILVANO, 2018).

\section{DISCUSSÃO}

Os estudos analisados nesta pesquisa apontam a necessidade do investimento na prevenção e promoção de saúde voltada a doenças crônicas no Brasil. Porque traz impactos positivos aos portadores e a sociedade. Visto que algumas intercorrências são situações que requerem atenção de urgência e de maior complexidade, desencadeiam hospitalizações, elevados custos aos cofres públicos e intenso sofrimento ao indivíduo e sua famílias e constituírem as principais causas de mortalidade no mundo (BARRETO et al, 2016).

Segundo Fernandez (2016) o programa carece de ações mais efetivas, para gerar mudança no comportamento dos usuários, uma vez que falta adesão às práticas propostas pelo programa, que desafia a atuação dos profissionais e da equipe. $O$ modelo de atenção vigente é precário e se torna um desafio aos gestores no enfrentamento das doenças crônicas.

A fisioterapia atua nos três níveis de atenção à saúde, sua prática se manteve reconhecida apenas aos níveis secundário e terciário de atenção à saúde. Existe uma carência de estudos sobre a atuação do fisioterapeuta na atenção básica e dos processos de estruturação da fisioterapia no nível primário de atenção à saúde. A inserção de profissional neste âmbito tem o olhar voltado ao cuidado da manutenção e garantia do movimento com qualidade. O fisioterapeuta pode e deve atuar nos três níveis de atenção à saúde, não se restringindo apenas à reabilitação, mas desenvolvendo uma prática integral e interdisciplinar de saúde (SALES, 2016).

Quase todos os artigos constatam que a participação do fisioterapeuta, pode ser benéfica, contribuindo com um olhar diferenciado, integral, uma vez que compreende o indivíduo como um ser funcional, em conjunto a equipe multidisciplinar, promovendo assim um atendimento mais completo às necessidades apresentadas pelos usuários do programa hiperdia (SALES, 2016).

No estudo realizado por Neves (2011), mostra que, apesar dos esforços, a fisioterapia ainda não possui uma definição clara em relação a sua atuação no programa. Dessa forma, é necessário debates sobre a atuação do fisioterapeuta nesse contexto, construindo para uma proposta mais específica, com diretrizes de atuação profissional uniformizadas na atuação básica nacional do fisioterapeuta.

Assim, fica evidente que os dados presentes na literatura não possibilitam analisar com precisão a função do fisioterapeuta e a necessidade de os profissionais dessa 
área serem mais envolvidos no processo de educação permanente, para que alcancem esta visão e ampliem seus métodos de atuação. As problematizações giram em torno das formas, das funções e dos significados de suas ocupações, sendo capaz de identificar como estas se relacionam com a saúde, o bem-estar e a qualidade de vida. Esse fato é o que torna suas ações, nos grupos, eficazes.

\section{CONSIDERAÇÕES FINAIS}

Diante da necessidade de um embasamento científico, guiando a uma práxis segura do profissional, ainda são escassos os estudos abordando a atuação do fisioterapeuta do programa de saúde pública Hiperdia. Os estudos encontrados demostram que há possibilidade de atuação e inserção do fisioterapeuta no programa e o mesmo, pode impactar de modo significativo na qualidade de vida dos usuários. Contudo é preciso enfatizar a necessidade de mais estudos abordando o tema, visando esclarecer o papel do profissional fisioterapeuta, delineando suas atribuições em cada nível de atenção à saúde e desta forma, justificar a inserção do fisioterapeuta no âmbito de saúde pública.

\section{REFERÊNCIAS}

AMORIM SERPA, Eliane; DANTAS DE LIMA, Ana Carollyne; DORNELAS DA SILVA, Ângela Cristina. Terapia ocupacional e grupo hiperdia. Journal of Occupational Therapy / Cadernos Brasileiros de Terapia Ocupacional. 2018. Disponível em: <http://web.a.ebscohost.com/ehost/pdfviewer/pdfviewer?vid=1\&sid=cfe95ddbf85b-4f8a-91e2-45f655a07e5d\%40sdc-v-sessmgr02>. Acesso em: 1 abr. 2019.

AUGUSTO, Viviane Gontijo et al. Promoção de saúde em unidades básicas: análise das representações sociais dos usuários sobre a atuação da fisioterapia. Scielo. Divinópolis, $2009 . \quad$ Disponível em:<https://www.scielosp.org/article/csc/2011. v16suppl1/957-963/pt/>. Acesso em: 3 abr. 2019.

BARRETO, Mayckel da Silva et al. Não utilização de consultas de rotina na Atenção Básica por pessoas com hipertensão arterial. Scielo, 2016. Disponível em: <http://www.scielo.br/pdf/csc/v23n3/1413-8123-csc-23-03-0795.pdf>. Acesso em: 17 abr. 2019.

BATISTA SILVANO, vinicíus. A inserção do fisioterapeuta na estratégia da saúde da família (esf), na cidade de barroso-MG. UFJF. 2018. Disponível em: <http://hermes.cpd.ufjf.br:8080/jspui/bitstream/ufjf/8740/1/viniciusbatistasilvano.pdf>. Acesso em: 8 abr. 2019.

BISPO JÚNIOR, J.P. Fisioterapia e Saúde Coletiva: desafios e novas responsabilidades profissionais. Ciência e Saúde Coletiva [periódico na internet] (2007/Set). [Citado em 10/04/2019]. Está disponível em: <http://www.cienciaesaudecoletiva.com.br/artigos/fisioterapia-e-saude-coletivadesafios-e-novas-responsabilidades-profissionais/1169?id=116> Acesso em: 3 abr. 2019. 
CEZÁRIO ALVES JÚNIOR, Ailton. Consolidando a rede de atenção às condições crônicas: experiência da rede hiperdia de minas gerais. organização pan-americana da saúde / organização mundial da saúde. Brasília, 2011. Disponível em:<http://bvsms.saude.gov.br/bvs/publicacoes/consolidando_rede_atencao.pdf $>$. A cesso em: 1 abr. 2019.

FERNANDEZ, Darla Lusia Ropelato et al. Programa hiperdia e suas repercussões sobre os usuários. Revista Baiana de Enfermagem. Salvador, v. 30, 11 jul. 2016. Disponível em:<HTTPS://portalseer. ufba.br/index.php/enfermagem/article/view/17156>. Acesso em: 10 abr. 2019.

GOMES, Ângela. Programa Hiperdia. Ministério da Saúde. Disponível em:<https://www.minsaude.gov.cv/index. php/documentosite/eventos/encontro-detrabalho-do-ministerio-da-saude-e-da-seguranca-social-realiza-durante-estasemana-de-8-a-12-de-agosto-de-2016-1/387-angelagomesap-hiperdia-cvelha/file>. Acesso em: 20 mar. 2019.

HIPERDIA. Portal da Saúde. DATASUS. Disponível em: $<$ http://datasus.saude.gov.br/sistemas-e-

aplicativos/epidemiologicos/hiperdia>. Acesso em: 26 mar. 2019.

HIPERDIA: Sistema de Cadastramento e Acompanhamento de Hipertensos e Diabéticos. DATASUS. Disponível em: <http://datasus.saude.gov.br/sistemas-eaplicativos/epidemiologicos/hiperdia>. Acesso em: 20 mar. 2019.

IBGE. Doenças crônicas atingem quase um terço da população brasileira. Agência IBGE Notícias. Disponível em:<https://agenciadenoticias. ibge.gov.br/agencia-salade-imprensa/2013-agencia-de-noticias/releases/12941-asi-doencas-cronicasatingem-quase-um-terco-da-populacao-brasileira>. Acesso em: 1 abr. 2019.

MINISTÉRIO DA SAÚDE. Cadernos de Atenção Básica: Caderno 7. Brasília, 2001. Disponível em: <http://bvsms.saude.gov.br/bvs/publicacoes/cd05_06.pdf>. Acesso em: 26 mar. 2019.

MINISTÉRIO DA SAÚDE. Diretrizes e Recomendações para o Cuidado Integral de Doenças Crônicas Não-Transmissíveis: Promoção da Saúde, Vigilância, Prevenção e Assistência. Brasília - $\quad$ DF, 2008. Disponível em:<http://bvsms.saude.gov.br/bvs/publicacoes/diretrizes_recomendacoes_cuidado_ doencas_cronicas.pdf>. Acesso em: 26 mar. 2019.

NEVES, Laura Maria Tomazi; ACIOLE, Giovanni Gurgel. Desafios da integralidade: revisitando as concepções sobre o papel do fisioterapeuta na equipe de Saúde da Família. Interface - Comunicação, Saúde, Educação, v. 15, p. 55, jun. 2011. Disponível em:<http://www.scielo.br/pdf/icse/2011 nahead/aop1011>. Acesso em: 17 abr. 2019. 
NOGUEIRA, Paula Roberta. plano de ação para o aumento e efetivação da participação dos usuários hipertensos e diabéticos ás reuniões do grupo operativo do hiperdia no município de piranga - MG. NESCON. Minas Gerais, 2013. Disponível em:<https://www.nescon.medicina.ufmg.br/biblioteca/imagem/4169.pdf>. Acesso em: 20 abr. 2019.

SALES, Raphaela Di Cavalcanti. O papel do fisioterapeuta residente multiprofissional em saúde da família: um relato de experiência. Revista de APS, v. 19, 2016. Disponível

em:<http://ojs2.ufjf.emnuvens.com.br/aps/article/view/15451/8125>. Acesso em: 10 abr. 2019.

SUS. Portal Principal de Noticias da Saúde. Disponível em:<http://portalms.saude.gov.br/sistema-unico-de-saude>. Acesso em: 19 mar. 2019.

SUS: Princípios e Conquistas. Ministério da Saúde. Brasília, 2000. Disponível em: <http://bvsms.saude.gov.br/bvs/publicacoes/sus_principios.pdf>. Acesso em: 19 mar. 2019.

SUS DE A A Z: Atendimento. FIOCRUZ. Disponível em:<https://pensesus. fiocruz.br/atendimento>. Acesso em: 20 mar. 2019. 